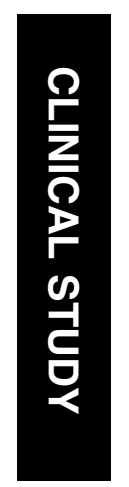

${ }^{1}$ Department of Ophthalmology, Taipei Medical University Hospital, Taipei, Taiwan

${ }^{2}$ Department of Ophthalmology, Taipei Medical University, Taipei, Taiwan

${ }^{3}$ School of Health Care Administration, Taipei Medical University, Taipei, Taiwan

${ }^{4}$ Department of Ophthalmology, Taipei City Hospital, Taipei, Taiwan

${ }^{5}$ Community Medicine Research Center, National Yang-Ming University, Taipei, Taiwan

Institute of Public Health, National Yang-Ming University, Taipei, Taiwan

${ }^{7}$ Department of Ophthalmology, National Taiwan University Hospital, Taipei, Taiwan

Correspondence: H-C Lin, School of Health Care Administration

Taipei Medical University, 250 Wu-Hsing Street,

Taipei 110 ,

Taiwan

Tel: + 886227361661

ext. 3613;

Fax: +886223789788

E-mail: henry11111@

tmu.edu.tw

Received: 7 November 2008 Accepted in revised form:

2 April 2009

Published online: 1 May 2009

Conflict of interest: None

\section{Surgeon age and operative outcomes for primary rhegmatogenous retinal detachment: a 3-year nationwide population-based study}

J-D Ho ${ }^{1,2}, \mathrm{~N}-\mathrm{W} \mathrm{Kuo}{ }^{3}, \mathrm{C}-\mathrm{Y}$ Tsai, ${ }^{4,5,6}, \mathrm{~S}-\mathrm{W}$ Liou $^{4,5,6,7}$ and $\mathrm{H}-\mathrm{C} \mathrm{Lin}^{3}$

$\leqslant 40-$, 41-to $50-$, and $\geqslant 51-$ year groups, respectively.

Conclusions Surgeon age is a relatively weak predictor for the outcomes of primary RRD surgery in aggregate in Taiwan and certainly not appropriate for discriminating performance among individual surgeons.

Eye (2010) 24, 290-296; doi:10.1038/eye.2009.99; published online 1 May 2009

Keywords: surgeon age; outcome;

rhegmatogenous retinal detachment; scleral buckling; pars plana vitrectomy

\section{(OR) of 180-day readmission between} ophthalmologist age groups were calculated.

Results For SB, patients treated by ophthalmologists aged $\geqslant 51$ years had a greater 180-day readmission rate $(O R=1.44,95 \%$ confidence interval $(\mathrm{CI})=\mathbf{1 . 0 1 - 2 . 0 7}, P<0.05$ ) than those treated by ophthalmologists aged 41-50 years. For PPV, patients treated by ophthalmologists aged $\leqslant 40$ years had a lower 180-day readmission rate than those treated by ophthalmologists aged $41-50$ and $\geqslant 51$ years $(\mathrm{OR}=0.58$ and $0.22,95 \% \mathrm{CI}=0.47-0.71$ and 0.13-0.63, $P<0.001$ and $<0.001$, respectively). For PPV + SB, patients treated by ophthalmologists aged $\geqslant 51$ years had a higher 180-day readmission rate than those treated by ophthalmologists aged $\leqslant 40$ and $41-50$ years $(\mathrm{OR}=3.32$ and $2.45,95 \% \mathrm{CI}=\mathbf{2 . 2 9 - 4 . 2 1}$ and 1.36-4.40, $P<0.01$ and $<0.01$, respectively). The absolute differences in the 180-day readmission rate by surgeon age ranged from -0.1 to $3.2 \%$. The proportions of selecting primary SB (without PPV) as the treatment modality were $33.1,35.6$, and $70.6 \%$ for the
Introduction

Medical advances occur frequently, and the knowledge that a physician possesses may easily become out of date. It has been shown that older physicians are less likely to incorporate new treatment strategies into their practice, prescribe appropriate regimens, and have a current knowledge base ${ }^{1-3}$ In a study examining the operative mortality utilizing national Medicare files of the United States, it was found that procedures performed by surgeons aged over 60 years resulted in higher mortality rates in pancreatectomy, coronary artery bypass grafting, and carotid endarterectomy compared to surgeons aged 41-50 years. For the other five procedures (elective aortic aneurysm repair, aortic valve replacement, oesophagectomy, lung resection, and cystectomy), there were no differences in the mortality rates between the surgeon group of $>60$ years and the surgeon group of 41-50 
years. In that study, patients treated by less-experienced surgeons (aged $\leqslant 40$ years) had comparable mortality rates to those treated by surgeons aged $41-50$ years for all eight procedures. ${ }^{4}$ Other studies also showed that more years of experience were associated with worse patient outcomes following carotid endarterectomy and coronary artery bypass surgery. ${ }^{5,6}$ On the other hand, it is generally assumed that the knowledge, skills, and experience accumulated by physicians during years of practice may result in better clinical capabilities. ${ }^{2}$ One report also suggested that surgeon youth and inexperience were more important risk factors for surgical mortality. ${ }^{7}$

To the best of our knowledge, no study has focused on the effects of surgeon age on operative outcomes of primary rhegmatogenous retinal detachment (RRD) surgery. In this study utilizing a nationwide populationbased administrative database, we examined the relationship between surgeon age and the 180-day readmission rate for primary RRD in Taiwan.

\section{Materials and methods}

\section{Database}

This study used pooled data for the years 2002-2004 obtained from the Taiwan National Health Insurance Research Database (NHIRD). The NHIRD covers all in-patient medical benefit claims for the Taiwanese population of over 22 million representing over $96 \%$ of the island's population. The database includes registries of contracted medical facilities and board-certified physicians, a monthly claims summary for in-patient claims, and details of in-patient orders. It provides operation procedure codes and diagnosis codes for each patient, using the International Classification of Disease, Ninth Revision, Clinical Modification (ICD-9-CM).

Because the study used secondary data without patient, institution, or physician identifiers, the ethics committee of the authors' institution granted waiver of informed consent for the study.

\section{Study sample}

The study sample was identified from the database by a principal admission diagnosis of retinal detachment and defects (ICD-9-CM codes 361-361.07) between January 2002 and December $2004(n=13382)$. We limited our study sample to those patients who underwent surgery consisting of scleral buckling (SB), pars plana vitrectomy $(\mathrm{PPV})$, or their combination $(\mathrm{PPV}+\mathrm{SB})(n=12042)$. We excluded cases undergoing primary pneumatic retinopexy in this study because of the small number of cases. In addition, we excluded those patients with a concomitant diagnosis of proliferative diabetic retinopathy (ICD-9-CM code 362.02) $(n=1370)$. We also excluded data on patients under 18 years of age $(n=421)$. Furthermore, because a patient may have had more than one operation for the treatment of retinal detachment, we only selected first-time admissions during the study period as the index hospitalization. Ultimately, our study sample comprised 7427 patients treated by 284 ophthalmologists.

\section{Variables of interest}

The key independent variable of interest was ophthalmologist age, whereas the key dependent variable was '180-day readmission,' with 'patient' as the unit of analysis. We divided ophthalmologists into three age groups: $\leqslant 40,41-50$, and $\geqslant 51$ years in accordance with prior studies. ${ }^{8,9}$ The '180-day readmission' was defined as a patient who was re-hospitalized with a principal diagnosis of RRD or tractional RD (ICD codes 361-361.07, 361.8, 361.81, 361.89, and 361.9), and undergoing surgery of SB, PPV, or PPV + SB within 180 days after the index admission.

\section{Statistical analysis}

The SAS statistical package (SAS System for Windows, version 8.2 ) was used. Global $\chi^{2}$-analyses were performed to examine the relationship between patient characteristics and ophthalmologist age group under different surgical modalities. Thereafter, separate multivariate logistic regression analyses employing generalized estimated equation (GEE) were utilized under different surgical modalities to assess the adjusted odds ratios (OR) of 180day readmission between ophthalmologist age groups after adjusting for patient age and gender, ophthalmologist gender and surgical volume for RRD in 2002-2004, and hospital level and volume of RRD in 2002-2004. Within the hospital level variable, each hospital was classified as a medical centre ( $>500$ beds), a regional hospital (251-500 beds), or a district hospital (21-250 beds).

The GEE method was also adopted as a means of accounting for any clustering of sampled patients among particular ophthalmologists. Clustering would indicate the greater likelihood for a given ophthalmologist's patient outcomes being similar to each other, as opposed to being similar to the patient outcomes of a different ophthalmologist. A two-sided $P$-value of $<0.05$ was considered statistically significant.

\section{Results}

The mean age of the 7427 patients was $49.8 \pm 15.9$ years. In total, 2837 (38.2\%) underwent SB, 2585 (34.8\%) PPV, 
and the remaining 2005 (27.0\%) both PPV and SB at the index hospitalization. Table 1 describes the demographic distribution of the sampled patients according to the surgical modality. The $\chi^{2}$-analyses showed that there were significant differences in patient age between surgical modalities $(P<0.001)$; patients who underwent SB had a greater tendency to be younger than those who underwent PPV or PPV + SB.

Table 2 provides the details of the demographic distributions of the sampled patients by ophthalmologist age group and surgical modality. For SB, patients treated by ophthalmologists of $\geqslant 51$ years were more likely to be older than patients treated by ophthalmologists in the other age groups $(P<0.001)$. However, no significant difference in patient age was observed between ophthalmologist age groups for PPV and PPV + SB. In addition, there were no significant differences in patient gender between ophthalmologist age groups for all different surgical modalities. The proportions of selecting primary SB (without PPV) as the treatment modality were 33.1, 35.6 , and $70.6 \%$ for the $\leqslant 40-, 41-$ to $50-$, and $\geqslant 51$-year groups, respectively. The oldest group of surgeons more frequently employed SB instead of PPV (with/without

Table 1 Demographic distribution of sampled patients undergoing surgery for primary RRD according to the surgical modality in Taiwan, 2002-2004

\begin{tabular}{|c|c|c|c|c|}
\hline \multirow[t]{2}{*}{ Variable } & \multicolumn{4}{|c|}{ Sampled patients } \\
\hline & Total, n (\%) & $S B, \mathrm{n}(\%)$ & $P P V, \mathrm{n}(\%)$ & $P P V+S B, \mathrm{n}(\%)$ \\
\hline Total & 7427 & 2837 & 2585 & 2005 \\
\hline \multicolumn{5}{|l|}{ Patient age (years) } \\
\hline$<50$ & $3285(44)$ & $1560(55)$ & $939(36)$ & 786 (39) \\
\hline $50-59$ & $1977(27)$ & $633(22)$ & $780(30)$ & $564(28)$ \\
\hline $60-69$ & $1371(19)$ & $436(16)$ & $544(21)$ & $391(20)$ \\
\hline$>69$ & $794(10)$ & $208(7)$ & $322(13)$ & $264(13)$ \\
\hline \multicolumn{5}{|l|}{ Patient gender } \\
\hline Male & $4514(61)$ & $1654(58)$ & $1598(62)$ & $1262(63)$ \\
\hline Female & $2913(39)$ & $1183(42)$ & $987(38)$ & $743(37)$ \\
\hline 180-Day readmission rate $(\%)$ & 15.8 & 13.4 & 15.6 & 17.8 \\
\hline Hospitalization day (mean \pm SD) & $5.9(3.9)$ & $5.6(3.4)$ & $5.8(4.0)$ & $6.7(4.3)$ \\
\hline Costs (mean \pm SD) (US\$) & $1433(538)$ & $1323(476)$ & $1330(508)$ & $1723(553)$ \\
\hline
\end{tabular}

PPV, pars plana vitrectomy; SB, scleral buckling.

Table 2 Demographic distribution of sampled patients undergoing surgery for primary RRD according to the surgical modality and ophthalmologist age group in Taiwan, 2002-2004

\begin{tabular}{|c|c|c|c|c|c|c|c|c|c|}
\hline \multirow[t]{4}{*}{ Variable } & \multicolumn{9}{|c|}{ Surgical modality } \\
\hline & \multicolumn{3}{|c|}{$S B, \mathrm{n}(\%)$} & \multicolumn{3}{|c|}{$P P V, \mathrm{n}(\%)$} & \multicolumn{3}{|c|}{$P P V+S B, \mathrm{n}(\%)$} \\
\hline & \multicolumn{3}{|c|}{ Ophthalmologist age group } & \multicolumn{3}{|c|}{ Ophthalmologist age group } & \multicolumn{3}{|c|}{ Ophthalmologist age group } \\
\hline & $\leqslant 40$ & $41-50$ & $\geqslant 51$ & $\leqslant 40$ & $41-50$ & $\geqslant 51$ & $\leqslant 40$ & $41-50$ & $\geqslant 51$ \\
\hline Total & 992 & 1305 & 540 & 1018 & 1408 & 159 & 984 & 955 & 66 \\
\hline \multicolumn{10}{|l|}{ Patient age (years) } \\
\hline$<50$ & $556(56)$ & $742(57)$ & $262(49)$ & $388(38)$ & $503(36)$ & $48(30)$ & $400(41)$ & $362(38)$ & $24(36)$ \\
\hline $50-59$ & $210(21)$ & $280(21)$ & $143(26)$ & $293(29)$ & $434(31)$ & $53(33)$ & $254(26)$ & $292(31)$ & $18(27)$ \\
\hline $60-69$ & $154(16)$ & $190(15)$ & 92 (17) & $222(22)$ & $288(20)$ & $34(22)$ & $191(19)$ & $187(20)$ & $13(20)$ \\
\hline$>69$ & $72(7)$ & $93(7)$ & $43(8)$ & $115(11)$ & $183(13)$ & $24(15)$ & $139(14)$ & $114(12)$ & $11(17)$ \\
\hline \multicolumn{10}{|l|}{ Patient gender } \\
\hline Male & $566(57)$ & $759(58)$ & $329(61)$ & $644(63)$ & $860(61)$ & $94(59)$ & $630(64)$ & $597(63)$ & $35(53)$ \\
\hline Female & $426(43)$ & $546(42)$ & $211(39)$ & $374(37)$ & $548(39)$ & $65(41)$ & $354(36)$ & $358(37)$ & $31(47)$ \\
\hline 180-Day readmission rate (\%) & 14.9 & 11.6 & 14.8 & 14.0 & 15.4 & 16.1 & 17.3 & 17.1 & 20.2 \\
\hline Hospitalization day (mean $\pm \mathrm{SD}$ ) & $5.1(2.9)$ & $5.6(3.0)$ & $6.3(4.8)$ & $5.7(3.4)$ & $5.7(3.8)$ & $7.2(7.7)$ & $6.7(3.9)$ & $6.4(3.9)$ & $10.8(10.1)$ \\
\hline
\end{tabular}

PPV, pars plana vitrectomy; SB, scleral buckling. 
SB) than did the $\leqslant 40$ - and 41 - to 50-year groups (both $P<0.001$ ).

Table 3 presents the adjusted OR for 180-day readmission by ophthalmologist age group under different surgical modalities. It is evident that patients treated by older surgeons had either a higher or similar 180-day readmission rate than those treated by younger surgeons, depending on the surgical modalities employed. For SB, patients treated by ophthalmologists of $\geqslant 51$ years had a greater 180-day readmission rate $(\mathrm{OR}=1.44,95 \%$ confidence interval $(\mathrm{CI})=1.01-2.07$, $P<0.05)$ than those treated by ophthalmologists of 41-50 years. For PPV, compared with patients treated by ophthalmologists of 41-50 years, patients treated by ophthalmologists of $\leqslant 40$ years had a smaller 180-day readmission rate $(\mathrm{OR}=0.58,95 \% \mathrm{CI}=0.47-0.71$, $P<0.001)$ after adjusting for other factors; when compared with patients treated by ophthalmologists of $\geqslant 51$ years, patients treated by ophthalmologists of $\leqslant 40$ years had a smaller 180-day readmission rate $(\mathrm{OR}=0.22$, $95 \% \mathrm{CI}=0.13-0.63, P<0.001)$ after adjusting for other factors. In addition, for PPV $+\mathrm{SB}$, after adjusting for other factors, the OR of 180-day readmission were 2.45 (95\% CI $=1.36-4.40, P<0.01$ ) times greater for patients treated by ophthalmologists of $\geqslant 51$ years than for patients treated by ophthalmologists of $41-50$ years; when compared with patients treated by ophthalmologists of $\leqslant 40$ years, patients treated by ophthalmologists of $\geqslant 51$ years age group had a greater 180-day readmission rate $(\mathrm{OR}=3.32,95 \% \mathrm{CI}=2.29-4.21$, $P<0.01)$ after adjusting for other factors. Regardless of the surgical modalities employed and using patients treated by surgeons aged $\leqslant 40$ years as the reference group, patients operated on by surgeons of 41-50 and $\geqslant 51$ years showed significantly higher overall 180-day readmission rates than those treated by surgeons aged $\leqslant 40$ years $(\mathrm{OR}=1.48$ and $1.57,95 \% \mathrm{CI}=1.14-1.92$ and $1.05-2.35, P<0.01$ and $<0.05$, respectively) after adjusting for patients' and providers' characteristics.

Table 3 shows surgeon gender differences for all procedures with female surgeons exhibiting a higher 180-day readmission rate. It is also evident in Table 3

Table 3 Adjusted OR and 95\% CI of 180-day readmission by ophthalmologist age group, 2002-2004

\begin{tabular}{|c|c|c|c|c|c|c|}
\hline \multirow[t]{3}{*}{ Variable } & \multicolumn{6}{|c|}{ Surgical modality } \\
\hline & \multicolumn{2}{|c|}{$S B$} & \multicolumn{2}{|c|}{$P P V$} & \multicolumn{2}{|c|}{$P P V+S B$} \\
\hline & Adjusted OR & $95 \% C I$ & Adjusted $O R$ & $95 \% C I$ & Adjusted OR & $95 \% \mathrm{CI}$ \\
\hline \multicolumn{7}{|c|}{ Ophthalmologist age group (years) } \\
\hline$\leqslant 40$ & 1.23 & $0.94-1.61$ & $0.58^{* * *}$ & $0.47-0.71$ & 1.05 & $0.81-1.35$ \\
\hline 41-50 (reference group) & 1.00 & & 1.00 & & 1.00 & \\
\hline$\geqslant 51$ & $1.44^{*}$ & $1.01-2.07$ & 1.21 & $0.92-1.42$ & $2.45^{* *}$ & $1.36-4.40$ \\
\hline \multicolumn{7}{|l|}{ Ophthalmologist gender } \\
\hline Male & $0.52^{* *}$ & $0.38-0.71$ & $0.57^{* * *}$ & $0.46-0.70$ & $0.49^{* * *}$ & $0.37-4.40$ \\
\hline Female (reference group) & 1.00 & & 1.00 & & 1.00 & \\
\hline Ophthalmologist volume & 1.00 & $1.00-1.00$ & $1.00^{* *}$ & $1.00-1.00$ & $1.00^{* *}$ & $1.00-1.00$ \\
\hline \multicolumn{7}{|l|}{ Patient age (years) } \\
\hline$<50$ (reference group) & 1.00 & & 1.00 & & 1.00 & \\
\hline $50-59$ & 1.27 & $0.97-1.65$ & 0.87 & $0.71-1.06$ & $1.31^{*}$ & $1.00-1.72$ \\
\hline $60-69$ & 0.98 & $0.71-1.36$ & 0.94 & $0.76-1.17$ & 1.08 & $0.79-1.48$ \\
\hline$>69$ & 1.02 & $0.66-1.58$ & 0.83 & $0.63-1.08$ & 1.09 & $0.77-1.56$ \\
\hline \multicolumn{7}{|l|}{ Patient gender } \\
\hline Male & $1.37^{* *}$ & $1.09-1.72$ & 1.18 & $0.99-1.40$ & 1.19 & $0.94-1.50$ \\
\hline Female (reference group) & 1.00 & & 1.00 & & 1.00 & \\
\hline \multicolumn{7}{|l|}{ Hospital level } \\
\hline Medical centre & 1.02 & $0.23-4.46$ & $0.32^{*}$ & $0.11-0.92$ & $0.35^{* *}$ & $0.16-0.78$ \\
\hline Regional hospital & 1.31 & $0.29-5.83$ & $0.28^{*}$ & $0.10-0.85$ & $0.29^{* *}$ & $0.13-0.69$ \\
\hline District hospital & 1.00 & & 1.00 & & 1.00 & \\
\hline Hospital volume & $1.00^{* *}$ & $1.00-1.00$ & $1.00^{* * *}$ & $1.00-1.00$ & $1.00^{* *}$ & $1.00-1.00$ \\
\hline
\end{tabular}

PPV, pars plana vitrectomy; SB, scleral buckling.

${ }^{*} P<0.05 ;{ }^{* *} P<0.01 ;{ }^{* *} P<0.001$. 
that both medical centres ( $>500$ beds) and regional hospitals (251-500 beds) had significantly lower 180-day readmission rates for $\mathrm{PPV}(\mathrm{OR}=0.32$ and 0.28 , 95\% CI $=0.11-0.92$ and $0.10-0.85$, both $P<0.05)$ and $\mathrm{PPV}+\mathrm{SB}(\mathrm{OR}=0.35$ and $0.29,95 \% \mathrm{CI}=0.16-0.78$ and $0.13-0.69$, both $P<0.01)$ than did district hospitals (21-250 beds).

\section{Conclusion}

In this nationwide population-based study, we found that the oldest group of surgeons ( $\geqslant 51$ years) more frequently employed SB instead of PPV (with/without SB) as the treatment modality for primary RRD than their younger counterparts. In addition, patients treated with SB for primary RRD by ophthalmologists $\geqslant 51$ years had a significantly higher 180-day readmission rate than those treated by ophthalmologists aged $41-50$ years after adjusting for confounding factors. For PPV, compared with patients treated by ophthalmologists $41-50$ or $\geqslant 51$ years, those treated by ophthalmologists in the $\leqslant 40$-year age group had a smaller 180-day readmission rate. For $\mathrm{PPV}+\mathrm{SB}$, the 180-day readmission rate was greater for patients treated by ophthalmologists in the $\geqslant 51$-year age group than for those treated by ophthalmologists of $\leqslant 40$ or $41-50$ years. To summarize, older group of surgeons had either similar or worse outcomes compared to those by the younger group of surgeons, depending on the surgical modalities employed.

One of the strengths of our study is that our patient sample is population based and consecutive. It includes a large number of surgeons, patients, and hospitals. This allowed us to include patients whose retina had redetached after the index surgery and sought follow-up treatment from other ophthalmologists or other hospitals. We adjusted for the effects of surgeon volume, hospital volume, and hospital level when comparing surgical outcomes among surgeons of different age groups. Our results showed that differences in operative outcomes of primary RRD among surgeons of different age groups could not be attributed to differences in surgeon volume, hospital volume, or hospital level.

A literature review focusing on the association of physician age with quality of care found that $73 \%$ of studies reported decreasing performance with increasing years in practice for some or all outcomes assessed; 3\% reported that performance initially increased with increasing experience, peaked, and then decreased; $21 \%$ reported no association; $4 \%$ reported increasing performance with increasing years in practice for some or all outcomes. ${ }^{10}$ For studies specifically of surgical performance, there are also other reports showing that more years of experience are associated with worse patient outcomes following carotid endarterectomy, coronary artery bypass surgery, and pancreatectomy. ${ }^{4-6}$ In contrast, one study reported that worse outcomes were observed for patients of young surgeons undergoing high-complexity alimentary tract surgery. ${ }^{7}$

Although our study did not address the potential mechanisms responsible for the relationship between surgeon age and operative outcomes of primary RRD surgery, several possibilities might explain this effect. First, the main shift to sub-specialization (including retinal sub-specialization) in departments of ophthalmology of hospitals in Taiwan occurred in the 1980s. Nearly all ophthalmologists in resident-training hospitals in Taiwan practiced in their sub-specialty after 1990. Younger surgeons were trained under the sub-specialization system and therefore practiced their sub-specialty after training. Increased sub-specialization in the younger generation of ophthalmologists may be responsible for the better operative outcomes in younger surgeon groups. Several retrospective sub-regional audits in the UK showed a statistically significant improvement in the primary success rate for primary RRD surgery during the audit periods. ${ }^{11-13}$ Those studies concluded that increasing subspecialization was responsible for the improvement. Second, there have been considerable improvements in instrumentation and technologies for vitrectomy in recent years (eg, the wide-angle fundus viewing system, high-speed vitrector, more-delicate intraocular instruments). ${ }^{14}$ Perhaps the physicians' 'toolkits' are created during training and may not be updated regularly. ${ }^{15}$ In addition, most ophthalmologists in Taiwan leave medical centres after completing their resident training course. Access to the newer instruments by the older surgeons may be limited by the budget constraint of the institutions where they are practicing. Less familiarity with newer technologies may partially explain the less-frequent adoption of primary PPV (with/without $\mathrm{SB}$ ) as the surgical modality and the inferior operative outcomes of primary PPV (with/ without SB) in the oldest group of surgeons ( $\geqslant 51$ years) compared to the youngest group ( $\leqslant 40$ years). ${ }^{15,16}$ Third, conventionally, SB is employed for routine and simpler cases, and primary PPV is employed in morecomplicated cases. ${ }^{17,18}$ In another study, we conducted regarding the operative outcomes of primary RRD in Taiwan from 1997 to 2005, we found a significant trend toward more-frequently employing primary PPV (with/without SB) for the management of primary RRD. Primary PPV (with/without SB) was a primary procedure in $47.3 \%$ of cases in 1997. This rate rose significantly to $61.2 \%$ in 2005 . A significant decrease in the total 180-day readmission rate occurred from $18.95 \%$ in 1997 to $13.81 \%$ in 2005 . These rates also significantly decreased for each surgical modality (from 16.30 to 
$11.38 \%$ for SB, from 21.29 to $14.69 \%$ for PPV, and from 22.99 to $16.55 \%$ for PPV + SB).${ }^{19}$ Our study showed the concurrence of the shift to more-frequent PPV (with/ without SB) use for primary RRD and a significant decrease in the 180-day readmission rate in Taiwan. It was shown in current study that the oldest group of surgeons ( $\geqslant 51$ years) more frequently employed SB instead of PPV (with/without SB) than their younger counterparts. The significantly higher 180-day readmission rate for SB performed by the oldest surgeon group ( $\geqslant 51$ years) may be partially due to their assigning more 'medium-complicated' RRD cases to primary SB surgery, whereas younger surgeons assigned only the 'simplest' RRD cases to primary SB surgery. On the other hand, RRD cases treated by PPV (with/without SB) by the oldest surgeons would be the 'mostcomplicated' cases, whereas RRD cases treated by younger surgeons included both 'medium-complicated' and the 'most-complicated' cases. This may partially explain the higher 180-day readmission rate for PPV performed by the oldest group of surgeons. Fourth, the fine motor skills and visual acuity necessary for RRD surgery may be diminished in some of the older surgeons. Finally, older surgeons may be more likely to delegate aspects of the procedure and peri-operative care to trainees. However, this might not explain the findings of our study because we have adjusted for the hospital level. Although there are many theories attempting to explain the effect of surgeon age on operative outcomes, elucidation of underlying mechanisms awaits additional studies.

We found in this study that female surgeons had a higher 180-day readmission rate for all procedures. However, because the number of female surgeons was relatively small ( $n=45,15.8 \%$ of the number of the surgeons), further study is needed to clarify if this was a chance finding. We also found that both medical centres (>500 beds) and regional hospitals (251-500 beds) had significantly lower 180-day readmission rates for PPV and PPV + SB than did district hospitals (21-250 beds). Budgets of smaller hospitals to purchase superior machines and/or instruments for PPV may be more limited than those of larger hospitals. This may be partially responsible for the significantly lower 180-day readmission rate after PPV and PPV + SB for medical centres and regional hospitals compared to district hospitals.

The findings of this study need to be interpreted in the context of the following limitations. First, the age-related differences in operative outcomes were possibly due to differences in patients' baseline risk. In our study, we adjusted for patient age and gender. However, other patient characteristics, such as chronicity of the disease or extent of the retinal detachment, were not available in the administrative database. Although we cannot rule out confounding by unmeasured variables in our analysis, there is no evidence from other studies that older surgeons systematically operate on patients with higher risk. Second, we used the 180-day readmission rate as an indicator of the primary failure rate. A similar definition of primary success/failure was adopted by another study. ${ }^{20}$ There must have been some patients with primary surgical failure (redetachment) who chose not to undergo the reoperation. Given the negligible barriers to medical access in the Taiwan National Health Insurance system and the serious visual impairment of untreated recurrent retinal detachment, we believe that the great majority of patients with redetachment after primary RRD repair would have selected to have the reoperation soon after onset of redetachment. Third, it is possible that readmission within 180 days after the index surgery for RRD is linked to the RRD of the contralateral eye because our analysis does not identify the eye undergoing the procedure. It would be rare for a patient to experience the occurrence of RRD in both eyes within 180 days. We believe that the presence of such rare cases would only minimally reduce the power of the analysis. Fourth, the outcomes observed in this study are most likely highly dependent on the medical system present in Taiwan and are not necessarily generalizable to other countries with different systems. Details as to the level of training of the 284 surgeons would be helpful; but these are not available in our database. However, our results suggest that older surgeons may need quality improvement interventions that are generally applicable to all surgeons. Finally, it must be stressed that the absolute differences in the 180-day readmission rate by surgeon age ranged from -0.1 to $3.2 \%$. This is a measure of 'clinical relevance' rather than statistical significance and should be emphasized. In addition, for SB, there was no significant difference in the 180-day readmission rate between the $\geqslant 51$ - and $\leqslant 40$-year groups. For PPV, there was no significant difference in the 180-day readmission rate between the $\geqslant 51$ - and 41 - to 50 -year groups.

Regarding the small absolute differences in the 180-day readmission rate by surgeon age, surgeon age should not be a primary factor in choosing an ophthalmologist for a patient facing the primary RRD surgery. Surgeon age is a relatively weak predictor for the outcomes of primary RRD surgery in aggregate and certainly much worse for discriminating performance among individual surgeon. On the other hand, certain surgeon characteristics may be more important, including risk-adjusted outcome measures when reliable and available, and possibly, other less-quantitative factors, such as surgeons' local reputation. 


\section{Acknowledgements}

This study is based in part on data from the National Health Insurance Research Database provided by the Bureau of National Health Insurance, Department of Health and managed by National Health Research Institutes. The interpretation and conclusions contained herein do not represent those of Bureau of National Health Insurance, Department of Health or National Health Research Institutes.

\section{References}

1 Stolley PD, Becker MH, Lasagna L, McEvilla JD, Sloane LM. The relationship between physician characteristics and prescribing appropriateness. Med Care 1972; 10: 17-28.

2 Rhee SO. Factors determining the quality of physician performance in patient care. Med Care 1976; 14: 733-750.

3 Ramsey PG, Carline JD, Inui TS, Larson EB, LoGerfo JP, Norcini JJ et al. Changes over time in the knowledge base of practicing internists. JAMA 1991; 266: 1103-1107.

4 Waljee JF, Greenfield LJ, Dimick JB, Birkmeyer JD. Surgeon age and operative mortality in the United States. Ann Surg 2006; 244: 353-362.

5 Hartz AJ, Kuhn EM, Pulido J. Prestige of training programs and experience of bypass surgeons as factors in adjusted patient mortality rates. Med Care 1999; 37: 93-103.

6 O’Neill L, Lanska DJ, Hartz A. Surgeon characteristics associated with mortality and morbidity following carotid endarterectomy. Neurology 2000; 55: 773-781.

7 Prystowsky JB. Are young surgeons competent to perform alimentary tract surgery? Arch Surg 2005; 140: 495-500.

8 Lien YC, Huang MT, Lin HC. Association between surgeon and hospital volume and in-hospital fatalities after lung cancer resections: the experience of an Asian country. Ann Thorac Surg 2007; 83: 1837-1843.
9 Lin HC, Xirasagar S, Lin HC, Hwang YT. Does physicians' case volume impact inpatient care costs for pneumonia cases? J Gen Intern Med 2008; 23: 304-309.

10 Choudhry NK, Fletcher RH, Soumerai SB. Systematic review: the relationship between clinical experience and quality of health care. Ann Intern Med 2005; 142: 260-273.

11 Ah-Fat FG, Sharma MC, Majid MA, McGalliard JN, Wong $D$. Trends in vitreoretinal surgery at a tertiary referral centre: 1987 to 1996. Br J Ophthalmol 1999; 83: 396-398.

12 Johnson Z, Ramsay A, Cottrell D, Mitchell K, Stannard K. Triple cycle audit of primary retinal detachment surgery. Eye 2003; 16: 513-518.

13 Comer MB, Newman DK, George ND, Martin KR, Tom BD, Moore AT. Who should manage primary retinal detachments? Eye 2000; 14: 572-578.

14 McLeod D. Is it time to call time on the scleral buckle? Br J Ophthalmol 2004; 88: 1357-1359.

15 Carthy P, Harvey I, Brawn R, Watkins C. A study of factors associated with cost and variation in prescribing among GPs. Fam Pract 2000; 17: 36-41.

16 Young MJ, Fried LS, Eisenberg J, Hershey J, Williams S. Do cardiologists have higher thresholds for recommending coronary arteriography than family physicians? Health Serv Res 1987; 22: 623-635.

17 Devenyi RG, de Carvalho Nakamura H. Combined scleral buckle and pars plana vitrectomy as a primary procedure for pseudophakic retinal detachments. Ophthalmic Surg Lasers 1999; 30: 615-618.

18 Ambresin A, Wolfensberger TJ, Bovey EH. Management of giant retinal tears with vitrectomy, internal tamponade, and peripheral 360 degrees retinal photocoagulation. Retina 2003; 23: 622-628.

19 Ho JD, Liou SW, Tsai CY, Tsai RJF, Lin HC. Trends and outcomes of treatment for primary rhegmatogenous retinal detachment: a 9-year nationwide population-based study. Eye 2009; 23(3): 669-675.

20 Sullivan PM, Luff AJ, Aylward GW. Results of primary retinal reattachment surgery: a prospective audit. Eye 1997; 11: 869-871. 